\title{
Indocyanine Green Nanoparticles Useful for Photomedicine
}

\author{
ANDERSON J. GOMES, ${ }^{1}$ LAURELÚCIA O. LUNARDI, ${ }^{2}$ JULIANA M. MARCHETTI, ${ }^{3}$ \\ CLAURE N. LUNARDI, ${ }^{3}$ and ANTONIO C. TEDESCO ${ }^{4}$
}

\begin{abstract}
Objective: The aim of this study was to evaluate the potential application of biodegradable nanoparticles (NPs) containing indocyanine green (ICG) in photodynamic therapy (PDT). Methods: Important parameters, such as particle size and external morphology, were established by dynamic light scattering (DLS) and scanning electron microscopy (SEM). Also, drug encapsulation efficiency and in vitro release behavior were evaluated by spectroscopic methods. Results: The particles are spherical in shape, they exhibit an 817-nm diameter, and they have a low tendency to aggregate. The loading efficiency was $65 \%$. ICG photophysical parameters showed a bathocromic shift in ICG-loaded nanoparticles (ICG-NP). Analysis of the cell P388-D1 in the presence of the ICG-NP by SEM showed that the majority of the nanoparticles were uptaken by phagocytic cells after $2 \mathrm{~h}$ of incubation. After laser irradiation photodamage was observed in P388-D1 cells where ICG-NPs had been uptaken by phagocytic cells. Conclusion: Polymeric NPs work as an efficient drug delivery system for PDT drugs, and this approach can be used in the administration of amphiphilic photosensitizers in the treatment of neoplasic cells.
\end{abstract}

\section{INTRODUCTION}

$\mathbf{P}$ HOTODYNAMIC THERAPY (PDT) is based on the use of photosensitizing chemicals that preferentially accumulate in the target tumor cells. ${ }^{1}$ The ideal PDT photosensitizer must be taken up by cells, and it must also absorb light at wavelengths that fall within the red region of the spectrum (650 and 800 $\mathrm{nm})$, known as a therapeutic window, where maximal penetration of light into tissues is observed. ${ }^{2}$ The recent interest in using indocyanine green (ICG) as a photosensitizer in PDT comes from the fact that this dye has a strong absorption band around $780 \mathrm{~nm}^{3}$, which falls in the "photodynamic window." 4,5 The ICG molecule exhibits a molecular structure with amphiphilic properties that has both hydrophilic and lipophilic properties $^{6,7}$ (Fig. 1).

The main disadvantage of using ICG in PDT is that it easily binds to $\alpha$-lipoprotein, a serum protein that is present in the plasma, which leads to the drug's rapid clearance from the body, mainly via the activity of the reticulum endothelial system
(RES). ${ }^{8,9}$ We proposed a strategy that will delay this clearance process, allowing efficient drug uptake. This strategy consists of using polymeric nanoparticles (NPs) formulated from the biodegradable polymer poly(DL-lactide-co-glycolide) (PLGA) as a controlled drug delivery system (DDS) for ICG in in vitro studies with the macrophage cell line P388-D1. The photophysical parameters of ICG, both in solution and in the DDS, were characterized to determine if drug efficacy would be preserved. We demonstrate here that ICG-loaded nanoparticles (ICG-NP) are efficiently uptaken by phagocytic cells, leading to detectable cellular surface damage after laser light stimulation.

\section{METHODS}

\section{Preparation of nanoparticles}

NPs were prepared by a W/O/W double-emulsion technique. ${ }^{10}$ ICG $(50 \mu \mathrm{M})$ was emulsified in methylene chloride

\footnotetext{
${ }^{1}$ Laboratório de Fotoquímica, Instituto de Química, Universidade Federal de Uberlândia, Uberlândia, MG, Brazil.

2Instituto de Biociência, Universidade Estadual Júlio de Mesquita Filho, Rio Claro, SP, Brazil.

${ }^{3}$ Faculdade de Ciências Farmacêuticas de Ribeirão Preto, Ribeirão Preto, SP, Brazil.

${ }^{4}$ Faculdade de Filosofia Ciências e Letras de Ribeirão Preto, Ribeirão Preto, SP, Brazil.
} 


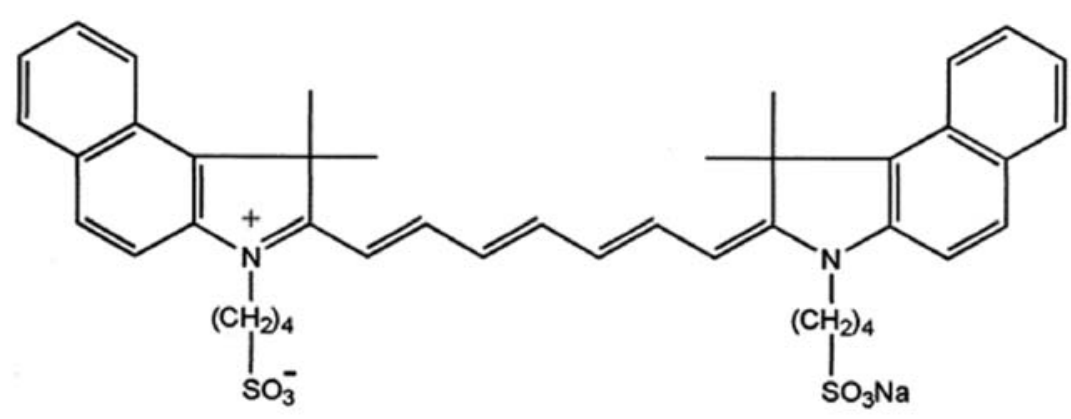

FIG. 1. Chemical structure of indocyanine green (ICG).
$(8.0 \mathrm{~mL})$ containing PLGA $(500 \mathrm{mg})$ using a homogenizer (Ultra-Turrax) for $60 \mathrm{sec}$ at $13,500 \mathrm{rpm}$. The primary W/O emulsion was transferred to $3.0 \%$ PVA $(100 \mathrm{~mL})$ and homogenized under ice cooling. Solvent evaporation was carried out by magnetic stirring at room temperature for $5 \mathrm{~h}$. NPs were recovered by centrifugation and washed (three times) with distilled water. The NP were lyophilized and stored in a desiccator. NPs without the dye were prepared by the same procedure.

\section{Drug entrapment efficiency (percentage)}

Aliquots of each aqueous sample with NPs $(2 \mathrm{~mL})$ had their absorbance at $780 \mathrm{~nm}$ read with a spectrophotometer (Hitachi U-3000). The drug content was indirectly estimated within the aqueous phase. The loading efficiency was calculated by Equation 1:

$$
\text { Loading efficiency }(\%)=\left(\frac{L_{A}}{L_{T}}\right) \times 100
$$

where $L_{A}$ is the amount of ICG in the NP, and $L_{T}$ is the total amount of drug (drug in the organic phase + drug in the aqueous phase).

\section{In vitro drug release}

NPs (10 mg) were placed into tubes and incubated in $10 \mathrm{~mL}$ of phosphate-buffered saline (PBS), $\mathrm{pH} 7.4$, under stirring at $37^{\circ} \mathrm{C}$. Every $24 \mathrm{~h}$, the NP suspension was centrifuged and the supernatant $(10 \mathrm{~mL})$ was withdrawn and replaced with medium $(10 \mathrm{~mL})$. This procedure was repeated for 7 days. The amount of ICG released was determined by spectrophotometric assay. The cumulative amount of ICG release was calculated by using Equation 2:

$$
\text { Cumulaive amount release }(\%)=\left(\frac{D_{T}}{D_{\infty}}\right) \times 100
$$

where $D_{t}$ is cumulative amount of drug release at time t, and $D_{\infty}$ is the total amount of drug release at time infinity.

\section{Particle size analysis}

Particle size and size distribution were determined in a dilute suspension $(1.0 \mathrm{mg} / \mathrm{mL})$ of NPs by dynamic light scattering (DLS) using equipment with a $10-\mathrm{mW} \mathrm{HeNe}$ laser beam with a wavelength of $633 \mathrm{~nm}$ and scattering angle of $90^{\circ}$.

\section{Spectroscopic measurements}

The absorption and fluorescence measurements were carried out with a Hitachi U3000 spectrophotometer and on a Hitachi F4500 spectrofluorimeter in quartz cuvettes of 1-cm length at $37^{\circ} \mathrm{C}$. The absorption spectrum was obtained for NPs-loaded ICG by using, as blanks, NP without ICG. The sample was excited at 780 and $790 \mathrm{~nm}$ (methanol and nanoparticle, respectively).

The fluorescence quantum yields $\left(\phi_{\mathrm{F}}\right)$ of ICG and NP were estimated from the corrected fluorescence spectra, using the secondary standard method. ${ }^{8}$ The fluorescence standard was zinc (II) phthalocyanine in toluene $\left(\phi_{\mathrm{F}}=0.30\right)$.

Singlet oxygen quantum yields were determined by using an indirect method described by Redmond and Gamlin. ${ }^{24}$ Irradiation was carried out with a diode array laser emitting at $805 \mathrm{~nm}$ (Optolaser). Light intensity was adjusted to $10^{15}$ photons $\mathrm{cm}^{-2} \mathrm{sec}^{-1}$. The DPBF absorption decay at $411 \mathrm{~nm}$ was detected after each irradiation cycle.

\section{Cell culture}

P388-D1 cells (American Type Culture Collection [ATCC] CCL46) were grown in $75-\mathrm{cm}^{2}$ flasks and maintained in Roswell Park Memorial Institute (RPMI) 1640 medium complemented with $10 \%$ fetal bovine serum (FBS). The cells were used in the growth logarithmic phase and cultured in a humidified incubator at $37^{\circ} \mathrm{C}$ with $5 \% \mathrm{CO}_{2}$.

For scanning electron microscopy (SEM) assay, macrophages were grown on glass pieces $(20 \times 20 \mathrm{~mm})$ and placed in Petri dishes. The cells were exposed to ICG-NP for $2 \mathrm{~h}$. SEM was carried out by the methodology described by Gomes et al. ${ }^{11}$ A similar procedure was used with NPs without dye after laser irradiation. Non-stimulated macrophages were used as controls.

\section{Dark toxicity assay}

The P388-D1 cells were seeded at a concentration of $0.5 \times$ $10^{6}$ cells in $2 \mathrm{~mL}$ per well of a 24 -well plate. They were incubated in the dark with photosensitizer solution $(50 \mu \mathrm{M})$ and dye-loaded NP $(0$ and $50 \mu \mathrm{M})$ at $37^{\circ} \mathrm{C}$, for $2 \mathrm{~h}$, with Hank's buffer. The cells were washed to remove excess dye and unbounded nanoparticle. An MTT solution $(150 \mu \mathrm{L})$ was added to each well, and dark toxicity was evaluated. ${ }^{11}$

\section{Phototoxicity assay}

The dose-dependent studies were determined by incubation of P388-D1 cells with ICG and NPs for $2 \mathrm{~h}$. The cells were ir- 
radiated with a fluency of $100 \mathrm{~J} / \mathrm{cm}^{2}$ using an array laser emitting at $805 \mathrm{~nm}$. After light exposure, the cells were incubated in RPMI 1640 for $24 \mathrm{~h}$, at $37^{\circ} \mathrm{C}$. Cell vitality was determined by MTT assay. ${ }^{11}$ Control cells were incubated for $2 \mathrm{~h}$ with RPMI 1640 without phenol red medium and irradiated under the same conditions.

\section{Statistical analysis}

Results are expressed as mean \pm SEM (standard error of the mean). Data were analyzed by one-way ANOVA (analysis of variance), followed by Newman-Keuls test for multiple comparisons. A $p$ value of $<0.05$ was considered to be statistically significant.

\section{RESULTS AND DISCUSSION}

The tendency of ICG to aggregate in solution is one of the main problems encountered in its use in PDT in physiological medium. This is because such an intermolecular association promotes non-radiative internal conversion, leading to a decrease in the excited triplet lifetime and to a reduction in the overall photosensitizing efficiency. ${ }^{12}$ The strategy used here to reduce ICG aggregation in aqueous medium involves the use of a DDS-in particular, NPs. The present study demonstrates that the double-emulsion method is a simple and suitable technique for the encapsulation of a water-soluble drug (ICG), with good reproducibility in relation to morphology, size distribution, encapsulation efficiency, and release profile. Figure 2 shows a representative micrograph of an ICG-NP, magnified at $25,000 \times$. For the experiments reported here, the NPs were spherical in shape and displayed a smooth surface, which can be seen from the external surface. No meaningful difference was found between ICG-NP and the lone NP, used as control.

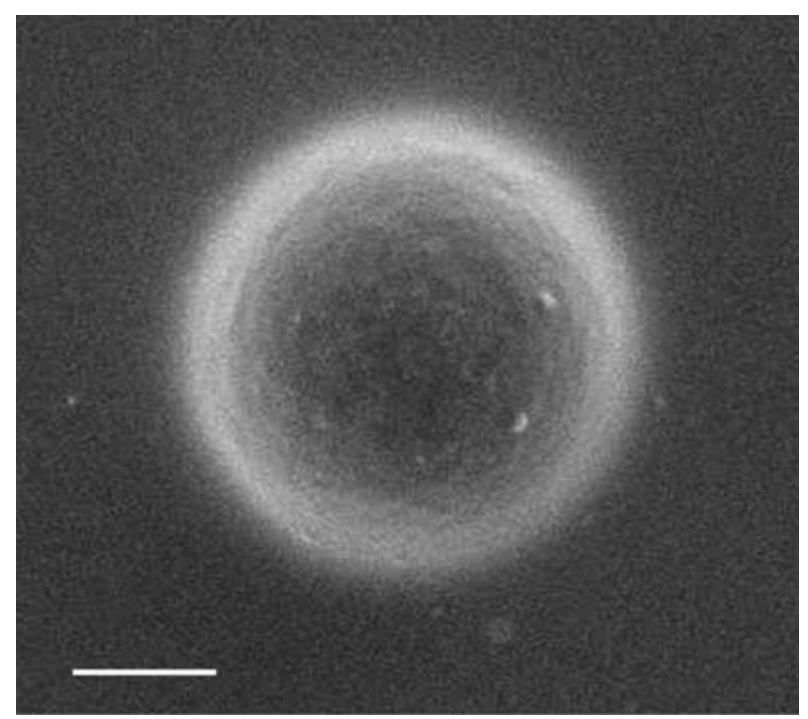

FIG. 2. Surface morphology of indocyanine green (ICG)-loaded nanoparticles prepared by the double emulsion method. Scanning electron microscopy, (SEM); original magnification, $\times 25,000$.
We have developed some modifications to the doubleemulsion method proposed by Zambaux. ${ }^{10}$ This modified method has led to smaller NPs, with a mean diameter of $817 \mathrm{~nm}$ $( \pm 70 \mathrm{~nm})$. ICG entrapment into the NPs did not significantly affect particle size. For the nanoparticle formulations used in this study, the polydispersivity index was $<0.049$, which indicates a narrow particle size distribution. Some studies with NPs have been undertaken in biomedical and biotechnological areas because of their small particle size $(10-1000 \mathrm{~nm})$, which is acceptable for intravenous (i.v.) injection. ${ }^{13}$ Smaller particles (diameter of several micrometers) seem to be inefficient as a DDS, because of their accumulation in lung capillaries and because they can be easily removed by the RES. ${ }^{14}$ In this way, our modification to the double-emulsion technique enables the preparation of particles, $<1.0 \mu \mathrm{m}$ in size, useful as DDS.

Drug-loading efficiency depends on factors such as the solubility of the photosensitizer in the solvents, and the physicochemical properties of the drug and the polymer. The improvement obtained in the efficiency of ICG encapsulation $(65 \pm 3 \%)$ is due to the increase in the concentration of PVA and PLGA when compared with the original proportions described by Zambaux et al. ${ }^{10}$ According to Kompella et al., ${ }^{15}$ PVA stabilizes the $\mathrm{O} / \mathrm{W}$ emulsion globules, thereby reducing diffusion of the drug into the aqueous medium. Saxena et al. have shown that PLGA NPs have a fixed capacity to entrap highly amphiphilic ICG molecules. ${ }^{16}$

The release of ICG into Hank's buffer was determined in order to obtain quantitative information about the profile of ICG release into the disperse system (7 days). Drugs formulated in polymeric devices are released either by diffusion through the polymer barrier or by erosion of the polymer material, or by a combination of both mechanisms. ${ }^{17}$ ICG-NP displays a biphasic pattern (Fig. 3).

The kinetics of drug release are important for the optimization of PDT therapy. The burst effect in the initial phase of drug release might be attributed to ICG molecules that are not entrapped but absorbed on the surface of the NP, or to ICG molecules that are accessible to the release medium through pores and channels formed during the NP preparation. This initial release profile reaches $54 \%$ after $24 \mathrm{~h}$. In the second phase (2-7 days), ICG released from the PLGA NP has been found to be slow due to the low porosity of the NPs, which means that ICG have to travel longer diffusion distances through the matrix. In this step, the exhaustion of ICG molecules near the surface of the NP is observed. ICG molecules completely entrapped in the PLGA matrix can only be released after the polymer matrix starts to lose its integrity. Such a combination of a burst release and a long-term release are ideal for the PDT protocol, allowing more than one lengthy application on alternate days without side effects for the patients.

\section{Photophysical properties}

The photophysical properties of ICG are shown in Table 1. ICG absorbs in the near-infrared part of the spectrum, which is important in melanoma treatment, because melanin is practically nonabsorbed in this region. Additionally, increased light penetration due to longer wavelength enhances treatment effectiveness. ${ }^{18}$ The maximum absorption for monomers in methanol occurs at $782 \mathrm{~nm}$. The encapsulation process shifts 


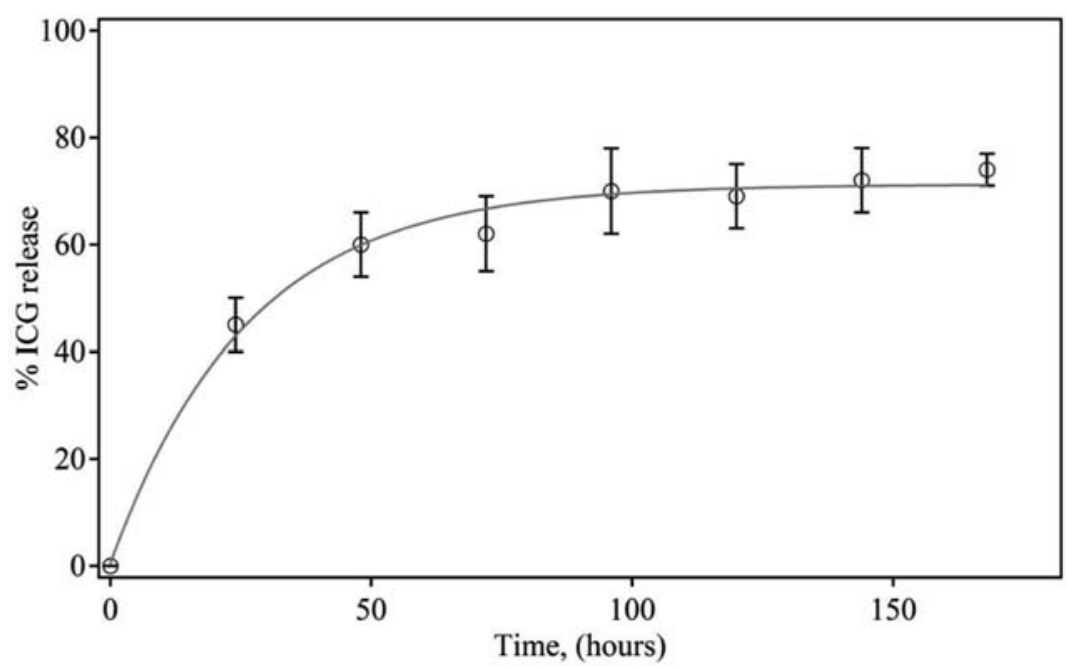

FIG. 3. Cumulative release profile of indocyanine green (ICG) from poly(DLlactide-co-glycolide) (PLGA) nanoparticle into Hank's buffer medium ( $\mathrm{pH}$ 7.4) after 7 days. the absorption band to $790 \mathrm{~nm}$. In the present study, when ICG was encapsulated in NPs, it had its absorption peak red-shifted by $8 \mathrm{~nm}$, compared with the spectrum of the monomer in methanol (Fig. 4). For emission spectra, ICG peaks appeared at $817 \mathrm{~nm}$ when the dye was in methanol. Encapsulation led to a 6-nm shifts of the ICG peak to $823 \mathrm{~nm}$ (Fig. 5).

Once the polymer backbone no longer absorbs in the visible region, the observed electronic transitions are due to monomeric ICG. Beems et al. ${ }^{19}$ attributed the absorption maximum to a $\pi \pi^{*}$ transition. The ICG fluorescence quantum yield $\left(\phi_{\mathrm{F}}\right)$ changes when the dye is adsorbed to macromolecules such as proteins and enzymes. In its non-bonded state, ICG is only weakly fluorescent, ${ }^{20}$ which we observed in our NP protocols. The fluorescence quantum yields of the monomer at low concentration are $0.04 \pm 0.004$ in methanol and $0.03 \pm 0.006$ in NP. Similar results have been obtained by Philip et al. ${ }^{21}$ in organic medium. The low monomer fluorescence quantum yields indicate dominant non-radiative $S_{1}-S_{0}$ relaxation (internal converting). Intersystem crossing (ISC) studies by Philip et al. ${ }^{21}$ indicate a small rate of $\phi_{\mathrm{S} \text { TT1 }}$ (methanol $=0.16$ ) and quantum yield of triplet oxygen formation, $\phi_{\mathrm{T}}<0.01^{3}$. Photodamage of cells after incubation with ICG solution is due, in part, to the formation of triplet oxygen and subsequent formation of singlet oxygen (reaction type II), but it is due, mainly, to free radical formation from excited states (reaction type I) and to electron transfer reactions starting from excited singlet or triplet states (reaction type III). ${ }^{22}$

The occurrence of photodynamic reactions of type II is somewhat surprising, as the quantum yield of ${ }^{1} \mathrm{O}_{2}$ generation $\left(\mathrm{F}_{\mathrm{D}}\right)$ is very low in homogeneous solution. ${ }^{23}$ So we decided to

Table 1. Spectroscopic and Photophysical Data OBtAINED FOR INDOCYANINE GREEN IN METHANOL AND NANOPARTICLES

\begin{tabular}{lccc}
\hline & $\lambda_{\max }, n m$ & $I F, n m$ & $\phi_{F}$ \\
\hline Methanol & 782 & 817 & $0.04( \pm 0.004)$ \\
Nanoparticle & 790 & 823 & $0.03( \pm 0.006)$ \\
\hline
\end{tabular}

employ the DPBF test to investigate the photochemical activity of ICG in methanol and NPs. Table 1 shows the results of the dye-sensitized photooxidation of DPBF. Values of singlet oxygen quantum yields for ICG were found to be 0.002 in both mediums studied in the present work. Similar results have been obtained by Redmond and Gamlin for ICG in solution. ${ }^{24}$ Considering these data, the cellular photodamage observed for ICG in methanol and NP may be due to the combined effects promoted by type I and type II mechanisms, with pronounced contribution from type II (low singlet oxygen quantum yield). The low singlet oxygen quantum yield is mainly due to the inefficient nature of the ISC process. Also, the fluorescence process competes with the deactivation process of the first excited singlet state. ${ }^{23}$

Despite these poor photophysical properties, ICG is able to produce powerful photosensitized cellular damage. ${ }^{6}$ To explain the antineoplasic activity of ICG, despite the low production of cytotoxic singlet oxygen, it has long been speculated that cellular dye localization and/or distribution may be important for the photobiological performance of a photosensitizer. ${ }^{25}$ ICG is cationic, in contrast with the more frequently used anionic photosensitizers such as hematoporphyrin derivatives, chlorines, and sulfonated phthalocyanine. Since a typical electric potential gradient of approximately $-180 \mathrm{mV}$ exists across the mitochondrial membrane, cationic cyanines strongly concentrate in the mitochondria, up to 1000 -fold with respect to the extracellular concentration. ${ }^{6}$

\section{Photobiological assays}

Our results support the hypothesis that PLGA NPs are a suitable delivery vehicle for ICG. Similar conclusions have been achieved by Tong et al. ${ }^{26}$ using PLGA microspheres as a delivery system for antitumor agents such as camptothecin.

In our study, the time effect was related to the incubation (30 min and $2 \mathrm{~h}$ ) of P388-D1 cells, with the ICG-NP evaluated by surface morphology of these cells using scanning electron micrographs. Figure 6A shows a micrograph of P388-D1 cells incubated with NPs for $30 \mathrm{~min}$; after this incubation time, a large quantity of NPs adhered to the cell surface. Figure 6B shows that P388-D1 cells incubated with NPs for $2 \mathrm{~h}$ display phago- 

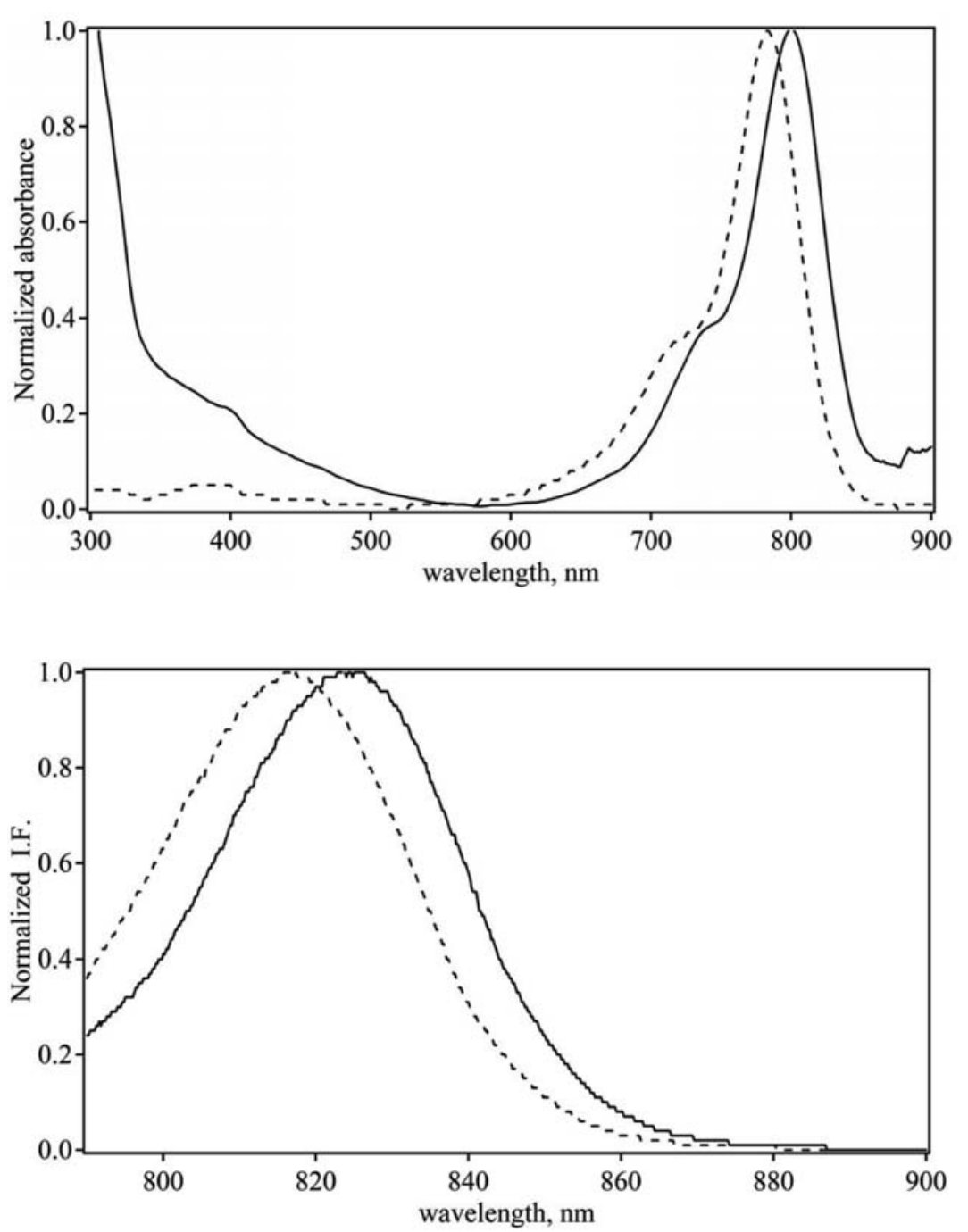

FIG. 4. Absorption spectra. --, Indocyanine green (ICG; $2.0 \mathrm{mM}$ ) in methanol; - , ICG-loaded nanoparticle.

FIG. 5. Emission spectra. --, Indocyanine green (ICG; $2.0 \mathrm{mM}$ ) in methanol; - ICG-loaded nanoparticle.
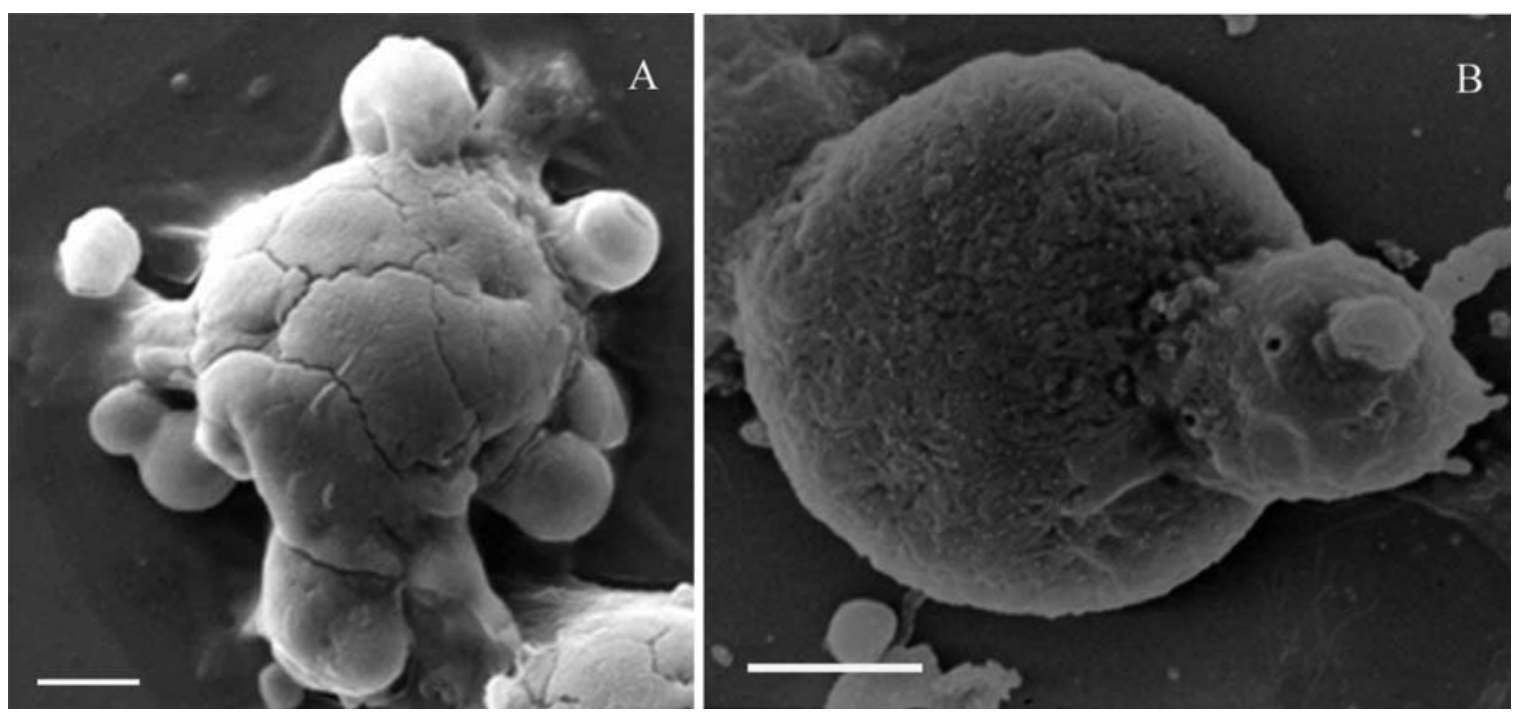

FIG. 6. Scanning electron micrograph of the macrophage P388-D1 cells exposed to indocyanine green (ICG)-loaded nanoparticles. (A) $30 \mathrm{~min}$ of incubation during the adhesion process. (B) $2 \mathrm{~h}$ of incubation, nanoparticle inside the cell in a phagocytosis process. Scale bar $=1 \mu \mathrm{m}$. 

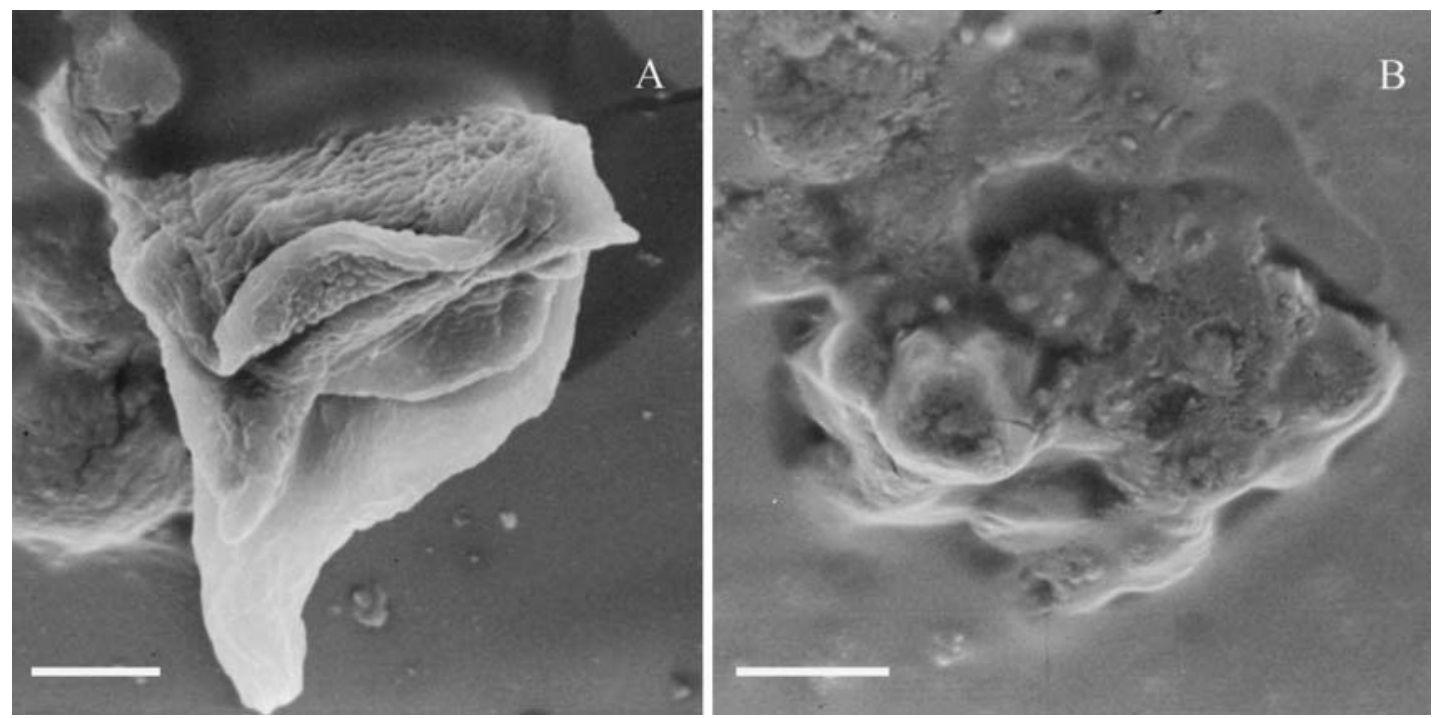

FIG. 7. (A) Scanning electron micrograph of macrophage P388-D1 cells exposed to indocyanine green (ICG) solution after a $100 \mathrm{~J} / \mathrm{cm}^{2}$ irradiation dose showing photodamage with cell morphology alteration. (B) P388-D1 cells exposed to ICG-loaded nanoparticles exposed to with $100 \mathrm{~J} / \mathrm{cm}^{2}$ irradiation showing the burst of the nanoparticles inside the cell. Scale bar $=1 \mu \mathrm{m}$.

cyted NPs, which can be noted by the fact that the cell appears as an extension of the cell surface.

These results indicate that the use of NPs as a DDS for photosensitizers in PDT needs to be critically established by means of size and morphology. These parameters must be strictly controlled to allow perfect cell uptake and biodistribution in the biological system.

After PDT, a sequence of photochemical and photobiological processes may lead to irreversible photodamage to the tumor cell. The results obtained in our studies (Fig. 7) indicate

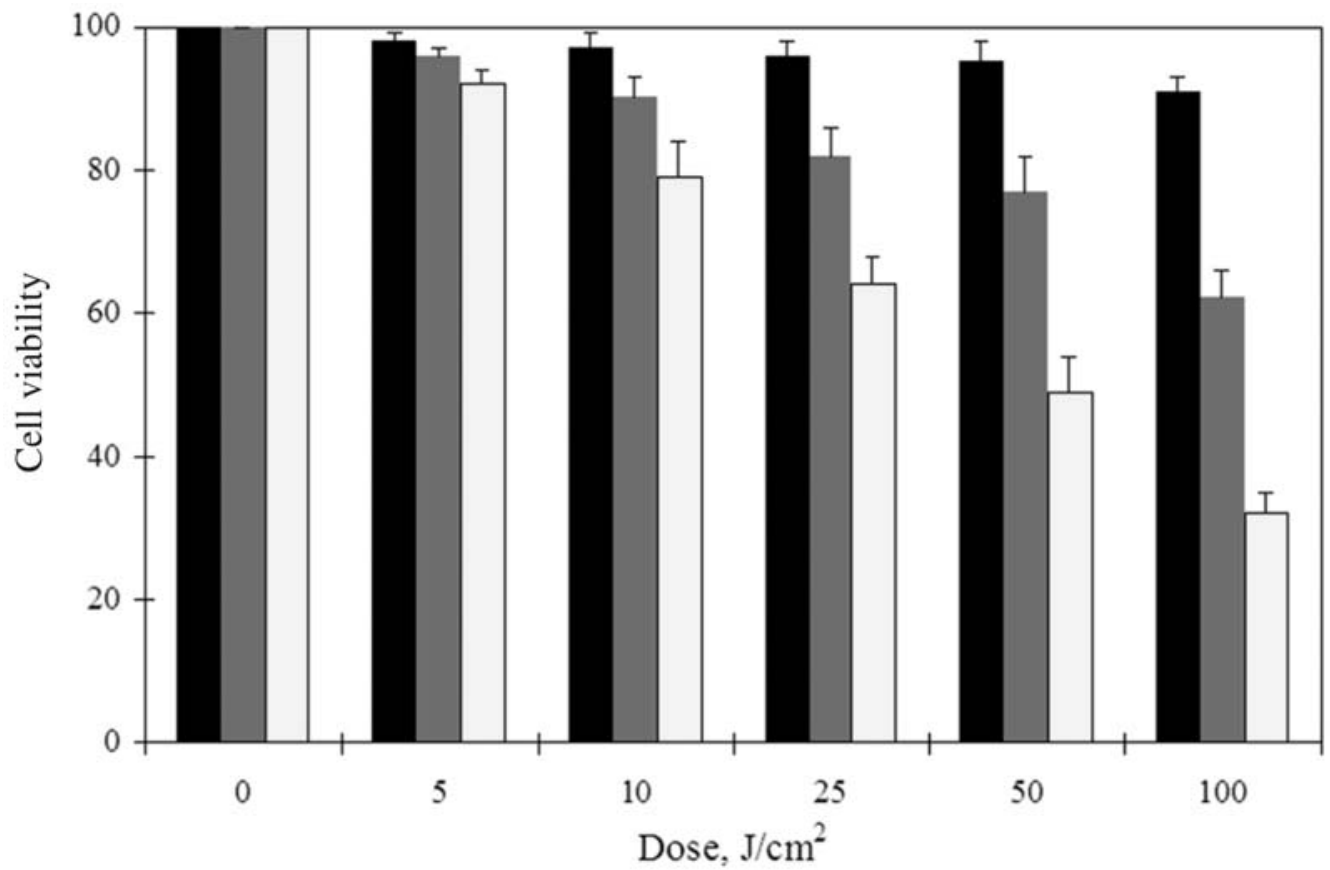

FIG. 8. Dose-dependent studies determined by incubation of P388-D1 cells with indocyanine green (ICG) $50 \mu \mathrm{M}$ in Hank's buffered solution $\mathrm{pH} 7.4(\square)$ and $50 \mu \mathrm{M}$ in the nanoparticle ( $\square$ ). Cell viability was determined by MTT assay. Control cells (匹) were incubated for $2 \mathrm{~h}$ with Hank's buffered solution $\mathrm{pH} 7.4$ and irradiated under the same conditions. Analysis of variance (ANOVA) showed that differences in the means were significant at any of the ICG and ICG-nanoparticle (NP) doses, $p<0.05$ (\# except for ICG-NP) at 5 and $10 \mathrm{Jcm}^{-2}$ ). 
the effect of the light dose on P388-D1 cell survival after $2 \mathrm{~h}$ of incubation with $50 \mu \mathrm{M}$ ICG in solution-that is, damage by rupture of the cell membrane, with loss of cytoplasmic material (Fig. 7A), - and the photodamage (Fig. 7B) of P388 cells incubated with ICG-NP, which occurs after irradiation with a fluency of $40 \mathrm{~J} / \mathrm{cm}^{-2}$. The uptake of the NP by the cells (without irradiation) leads to no cellular damage.

The MTT assay shows that both ICG in solution and the ICG-NP are able to mediate the photodynamic killing of P388D1 cells in a dose-dependent manner in relation to controls. Our results show that $\mathrm{LD}_{50}$ of ICG is $40 \mathrm{Jcm}^{-2}$ in the case P388D1 cells. In ICG-NP, a 23\% decrease in cell death is observed at the same laser dose (Fig. 8). The results are expressed as percentage of cell death. The ANOVA showed that differences in the means were significant at any of the ICG and ICG-NP doses, at $p<0.05$ (except for ICG-NP at 5 and $10 \mathrm{Jcm}^{-2}$ ).

\section{CONCLUSION}

Administration of drugs using biodegradable PLGA polymers has generated interest due to their biocompatibility and biodegradability. The method described in this work is suitable for the encapsulation of amphiphilic drugs such as ICG. The technique employed in the preparation of the NPs is reproducible, leading to drug incorporation of $65 \%$ and a narrow size distribution $(817 \pm 70 \mathrm{~nm})$. Photophysical parameters for ICG have been evaluated in homogeneous and in NP media, showing that this encapsulated system preserves the drug's properties. We demonstrated that ICG-NP took up macrophages after $2 \mathrm{~h}$ of incubation. Our results have also shown that phagocyted cells incubated with ICG-NP are susceptible to huge photodamage after irradiation, probably leading to a series of events triggered mainly by the local production of reactive oxygen species at specific sites inside the cell. These results reinforce the idea that the use of DDS (such as PLGA NPs) as carriers for photosensitizers is useful for PDT of neoplasic tissues.

\section{ACKNOWLEDGMENTS}

We would like to thank Dr. Flávio Henrique from the Electron Microscopy Center of the Instituto de Biociências UNESP-Rio Claro. We gratefully acknowledge FAPESP for financial support A.J.G. grant 98/11712-0 and C.N.L. grant 00/00431-1.

\section{REFERENCES}

1. Costa, R.A., Farah, M.E., Freymuller, E., et al. (2001). Choriocapillaris photodynamic therapy using indocyanine green. Am. J. Ophthalmol. 132, 557-565.

2. Grahn, M.F., McGuinnes, A., Benzie, R., et al. (1997). Intracellular uptake, absorption spectrum and stability of the bacteriochlorin photosensitizer 5,10,15,20-tetrakis(m-hydroxyphenyl)bacteriochlorin (mTHPBC). Comparison with 5,10,15,20-tetrakis(mhydroxyphenyl)chlorin (MTHPC). J. Photochem. Photobiol. B 37, 261-266.
3. Reindl, S., Penzofer, A., Gong, S.-H., et al. (1997). Quantum yield of triplet formation for indocyanine green. J. Photochem. Photobiol. A Chem. 105, 65-68.

4. Borland, C.F., McGarvey, D.J., and Truscott, T.G. (1987). Photophysical studies of bacteriochlorophyll-a and bacteriopheophytina-singlet oxygen generation. J. Photochem. Photobiol. B Biol. 1, 93-101.

5. Henderson, B.W., Sumlin, A.B., Owczarczak, B.L., et al. (1991). Bacterichlorophyll a as photosensitizer for photodynamic treatment of transpantable murine tumors. J. Photochem. Photobiol. B. Biol. 10, 303-313.

6. Delaey, E., van Laar, F., De Vos, D., et al. (2000). A comparative study of the photosensitizing characteristics of some cyanine dyes. J. Phys. Chem. B 55, 27-36.

7. Desmetre, T., Devoisselle, J.M., and Mordon, S. (2000). Fluorescence properties and metabolic features of indocyanine green (ICG) as related to angiography. Surv. Ophthalmol. 45, 15-27.

8. Abels, C., Karrer, S., Bäumler, W., et al. (1998). Indocyanine green and laser light for the treatment of AIDS-associated cutaneous Kaposi's sarcoma. Br. J. Cancer 77, 1021-1024.

9. Saxena, V., Sadoqi, M., and Shao, J. (2004). Enhanced photo-stability, thermal-stability and aqueous-stability of indocyanine green in polymeric nanoparticulate systems. J. Photochem. Photobiol. B Biol. 74, 29-38.

10. Zambaux, M.F., Bonneaux, F., Gref, R., et al. (1998). Influence of experimental parameters on the characteristics of poly(lactic acid) nanoparticles prepared by a double emulsion method. J. Control. Rel. 50, 31-40.

11. Gomes, A.J., Lunardi, L.O., Marchetti, J.M., et al. (2005). Photobiological and ultrastructural studies of nanoparticles of poly(lactic-co-glycolic acid)-containing bacteriochlorophyll-a as a photosensitizer useful for PDT treatment. Drug Deliv. 12, 159-164.

12. Fu, F., Li, X., and Wu, C. (2002). Encapsulation of phthalocyanines in biodegradable poly(sebacic anhydride) nanoparticles. Langmuir 18, 3843-3847.

13. Kreuter, J. (2004). Influence of the surface properties on nanoparticle-mediated transport of drugs to the brain. J. Nanosci. Nanotechnol. 4, 484-488.

14. Jeon, H.-J., Jeong, Y.-I., Jang, M.-K., et al. (2000). Effect of solvent on the preparation of surfactant-free poly(DL-lactide-coglycolide) nanoparticles and norfloxacin release characteristics. Int. J. Pharm. 207, 99-108.

15. Kompella, U.B., Bandi, N., and Ayalasomayajula, S.P. (2001). Poly(lactic acid) nanoparticles for sustained release of budesonide. Drug Deliv. Technol. 1, 28-34.

16. Saxena, V., Sadoqi, M., and Shao, J. (2004). Indocyanine green-loaded biodegradable nanoparticles: preparation, physicochemical characterization and in vitro release. Int. J. Pharm. 278, 293-301.

17. Dunne, M., Corrigan, O.I., and Ramtoola, Z. (2000). Influence of particle size and dissolution conditions on the degradation properties of polylactide-co-glycolide particles. Biomaterials 21, 16591668.

18. Urbanska, K., Romanowska-Dixon, B., Matuszak, Z., et al. (2002). Indocyanine green as a prospective sensitizer for photodynamic therapy of melanomas. Acta Biochim. Polon. 49, 387-391.

19. Beems, E.M., Dubbelman, T.M., Lugtenburg, J., et al. (1987). Photosensitizing properties of bacterichlorophyllin-a and bacteriochlorin-a, two derivatives of bacteriochlorophyll-a. Photchem. Photobiol. 46, 639-643.

20. Moody, E.D., Viskari, P.J., and Colyer, C.L. (1999). Non-covalent labeling of human serum albumin with indocyanine green: a study by capillary electrophoresis with diode laser-induced fluorescence detection. J. Chromatogr. B 729, 55-64. 
21. Philip, R., Penzofer, A., Bäumler, W., et al. (1996). Absorption and fluorescence spectroscopic investigation of indocyanine green. J. Photochem. Photobiol. A Chem. 96, 137-148.

22. Gratz, H., Penzkofer, A., Abels, C., et al. (1999). Photo-isomerisation, triplet formation, and photo-degradation dynamics of indocyanine green solutions. J. Photochem. Photobiol. A Chem. 128, 101-109.

23. Krieg, M., Bilitz, J.M., Srichai, M.B., et al. (1994). Effects of structural modifications on the photosensitizing properties of dialkylcarbocyanine dyes in homogeneous and heterogeneous solutions. Biochim. Biophys. Acta 1199, 149-156.

24. Redmond, R.W., and Gamlin, J.N. (1999). A compilation of singlet oxygen yields from biologically relevant molecules. Photochem. Photobiol. 70, 391-475.
25. Krieg, M., and Bilitz, J.M. (1996). Structurally modified trimethine thiacarbocyanine dyes - effect of $N$-alkyl substituents on antineoplastic behavior. Biochem. Pharmacol. 51, 1461-1467.

26. Tong, W., Wang, W., and D'Souza, M. (2003). Evaluation of PLGA microspheres as delivery system for antitumor agentcamptothecin. Drug Device Industrial Pharm. 29, 745-756.

Address reprint requests to:

Dr. Antonio Claudio Tedesco

Av. Bandeirantes 3900

Ribeirão Preto, SP, Brazil 14040-901

E-mail: tedesco@ffclrp.usp.br 